\title{
Systematic screen of potential circular RNA biomarkers of Hirschsprung's disease
}

\author{
Shun-Gen Huang ${ }^{1}$, Yuan Cheng ${ }^{1}$, Dashuang Li $^{1}$, Chao Sun ${ }^{1}$, Fang Fang ${ }^{2}$, Wan-Liang Guo ${ }^{3}$ \\ ${ }^{1}$ Pediatric Surgery, Children's Hospital of Soochow University, Suzhou, China; ${ }^{2}$ Institute of Pediatric Research, Children's Hospital of Soochow \\ University, Suzhou, China; ${ }^{3}$ Department of Radiology, Children's Hospital of Soochow University, Suzhou, China \\ Contributions: (I) Conception and design: WL Guo; (II) Administrative support: None; (III) Provision of study materials or patients: SG Huang; \\ (IV) Collection and assembly of data: Y Cheng, D Li; (V) Data analysis and interpretation: F Fang, C Sun, SG Huang; (VI) Manuscript writing: All \\ authors; (VII) Final approval of manuscript: All authors. \\ Correspondence to: Chao Sun. Pediatric Surgery, Children's Hospital of Soochow University, Suzhou 215025, China. Email: sunchao.0819@163.com; \\ Fang Fang. Institute of Pediatric Research, Children’s Hospital of Soochow University, Suzhou 215025, China. Email: baseff@sina.com; Wan-Liang \\ Guo. Department of Radiology, Children’s Hospital of Soochow University, Suzhou 215025, China. Email: gwlsuzhou@163.com.
}

Background: Hirschsprung's disease (HSCR) is a developmental disorder of the enteric nervous system in which enteric ganglia are missing along a portion of the intestine. Aberrant expression of several circular RNAs (circRNAs) has been identified in the disease, but the full range of dysregulated circRNAs and their potential roles in its pathogenesis remain unclear. We used microarray profiling to systematically screen for circRNAs that were differentially expressed in HSCR, and we comprehensively analyzed the potential circRNA-miRNA-mRNA regulatory network to identify molecular mechanisms involved in the disorder.

Methods: We identified circRNAs that were differentially expressed between diseased tissue and paired normal intestinal tissues from patients with HSCR. The most strongly upregulated circRNAs were then validated by quantitative reverse-transcription-PCR (RT-PCR). We also constructed a circRNA-miRNAmRNA interaction network to determine functional interactions between miRNAs and mRNAs.

Results: We identified 17 circRNAs that were upregulated and 10 that were downregulated in HSCR tissue compared with normal tissues. The five circRNAs that showed the greatest upregulation were verified by RT-PCR: hsa_circRNA_092493, hsa_circRNA_101965, hsa_circRNA_103118, hsa_circRNA_103279, and hsa_circRNA_104214. These five circRNAs were successfully adopted to diagnose HSCR based on receiver operating characteristic curves, and they were used to generate a circRNA-miRNA-mRNA network. The network revealed a potential function of the circRNAs as molecular sponges targeting miRNAs and mRNAs in HSCR.

Conclusions: This first-ever systematic dissection of the circRNA profile in HSCR may provide useful insights into improving diagnosis and therapy.

Keywords: Hirschsprung's disease (HSCR); circular RNA (circRNA); microarray expression profile; diagnosis

Submitted Aug 17, 2021. Accepted for publication Dec 22, 2021.

doi: 10.21037/tp-21-392

View this article at: https://dx.doi.org/10.21037/tp-21-392

^ ORCID: 0000-0003-3849-2904. 


\section{Introduction}

Hirschsprung's disease (HSCR) is a developmental disorder of the enteric nervous system in which ganglion cells are absent along the intestine from the myenteric and submucosal plexuses to the anorectum (1-4). Although the diagnosis and treatment of this disease have improved substantially $(5,6)$, early diagnosis remains a challenge (7). The techniques using barium enemas and pathological biopsy show high diagnostic accuracy, but they are invasive and possess their own potential risks, and some even need to be repeated to confirm an initial diagnosis (8). Noninvasive diagnostic methods such as testing of genetic material in peripheral blood might therefore facilitate early detection and timely intervention.

Potentially useful forms of genetic material in this regard are the circular RNAs (circRNAs), which are endogenous, noncoding RNAs expressed by many species at particular developmental stages and in particular tissues, and may therefore deliver the specificity needed to function as diagnostic and prognostic biomarkers (9). Dysregulation of circRNAs appears to contribute to many pathological processes, including cancer and diseases of the cardiovascular and nervous systems (9-12). Specifically, dysregulation of several circRNAs has been observed in HSCR $(13,14)$, and many non-coding RNAs appear to be dysregulated in this disorder $(15,16)$.

These observations raise the possibility of the noninvasive diagnosis and analysis of HSCR based on circRNAs due to their salient features, which include significant stability, high abundance, evolutionary conservation, and tissue-specific expression. A crucial first step in exploring this possibility is to understand the complete landscape of circRNAs, the expression of which is altered in the disease. Such work may also identify molecular pathways that malfunction in the disorder, and thus provide insights into guiding the development of novel therapies. With these goals in mind, in the present study, we performed microarray profiling to systematically identify potential circRNA biomarkers of HSCR, and we also examined the potential biological functions of these circRNAs in order to guide future research. We present the following article in accordance with the STARD reporting checklist (available at https:// tp.amegroups.com/article/view/10.21037/tp-21-392/rc).

\section{Methods}

\section{Study subjects}

The study was conducted in accordance with the Declaration of Helsinki (as revised in 2013). The study procedure was approved by the Ethics Committee of our Hospital (No. 20170606013) and written informed consent was obtained from the legal guardians of all of the participating children.

\section{$R N A$ extraction}

Megacolon and adjacent, paired normal intestinal tissues were biopsied from the same patient during surgery, and the tissues were stored at $-80{ }^{\circ} \mathrm{C}$ before RNA extraction with TRIzol reagent (Invitrogen, USA).

\section{Microarray analysis}

Microarray analyses were carried out by Shanghai KangChen Biotech (Shanghai, China) using a human circular RNA Array 2.0 (8x15K, Arraystar, Maryland, USA). Differentially expressed circRNAs were defined as those showing a fold-change of at least 1.2.

\section{Construction of a competing endogenous RNA (ceRNA) network}

The five circRNAs upregulated to the greatest extent in HSCR based on our microarray study were used to generate a network of interactions among the selected circRNAs, sponged microRNAs (miRNAs), and target mRNAs in Cytoscape software. The network was generated using a proprietary miRNA target-prediction software (Arraystar, USA) developed from TargetScan and miRanda $(17,18)$.

\section{Real-time reverse-transcription-PCR (RT-PCR)}

We executed real-time RT-PCR to validate that the five circRNAs found in our microarray analysis were the most strongly upregulated in HSCR. Reactions were performed using a $2 \times$ PCR Master Mix (Beyotime, Wuhan, China) and the primers are listed in Table 1. Relative expression was calculated using the $2^{-\Delta \Delta \mathrm{Ct}}$ method and normalized to the expression of $\beta$-actin.

\section{Statistical analysis}

We analyzed data statistically using SPSS 20.0 (IBM, Chicago, IL, USA). Differences in circRNA expression between HSCR tissue and paired normal intestinal tissues were assessed for significance using the Student's $t$-test. We assessed the ability of the five circRNAs that were most 
Table 1 Upstream and downstream primer sequences

\begin{tabular}{|c|c|c|c|}
\hline Gene name & Primer sequences & Annealing temperature $\left({ }^{\circ} \mathrm{C}\right)$ & Product length $(\mathrm{bp})$ \\
\hline$\beta$-actin & R: 5'-CCTGTAACAACGCATCTCATATT-3' & & \\
\hline \multirow[t]{2}{*}{ hsa_circRNA_101965 } & F: 5'-GCTACGATGGATGTGGACCTG-3' & 60 & 273 \\
\hline & R: 5'-GCTGTATTTCCGAAGCAAAGAGT-3' & & \\
\hline hsa_circRNA_092493 & R: 5'-ACTGTGTGTCAAACAAGGTGCTG-3' & & \\
\hline \multirow[t]{2}{*}{ hsa_circRNA_103118 } & F: 5'-TCAGAAATTGAGAAGCTGGTAA-3' & 60 & 61 \\
\hline & R: 5'-GAGACAGTGAAATTATCCGTTG-3' & & \\
\hline hsa_circRNA_103279 & F: 5'-GGCTGTGCCAGGCTTTTG-3' & 60 & 145 \\
\hline hsa_circRNA_104214 & R: 5'-GTCGGCTTTCTTTGATTTGAG-3' & & \\
\hline
\end{tabular}

strongly upregulated in HSCR to diagnose the disorder based on the area under the receiver operating characteristic (ROC) curve (AUC). All of the $\mathrm{P}$ values were two-sided, and differences associated with a $\mathrm{P}<0.05$ were considered to be statistically significant.

\section{Results}

\section{Demographic and clinical data}

We included diseased and normal tissues from four patients with pediatric congenital megacolon (median age, 4 months; interquartile range, 3-7 months) in the microarray. Demographic and clinical data are summarized in Table 2.

\section{Microarray analysis}

Microarray analysis identified 27 differentially expressed circRNAs, of which 17 were upregulated and 10 downregulated in HSCR (Figure 1). Samples of diseased tissue showed a relatively homogeneous pattern of circRNA expression, as did samples of normal tissue. The five circRNAs most strongly upregulated in HSCR were hsa_circRNA_092493, hsa_circRNA_101965, hsa_ circRNA_103118, hsa_circRNA_103279, and hsa_ circRNA_104214. We therefore focused on these circRNAs in the subsequent analyses described below.

\section{ceRNA analysis}

To begin to understand how the five most strongly upregulated circRNAs may contribute to HSCR, we generated a circRNA-miRNA-mRNA regulatory network for each circRNA (Figures 2-6). These networks suggested that each circRNA sponge certain miRNAs, thereby derepressing the mRNAs targeted by the miRNAs $(19,20)$.

\section{RT-PCR validation of circRNAs}

The five most strongly up-regulated circRNAs from the microarray analysis were verified by RT-PCR of biopsies taken from an independent set of 15 children with congenital megacolon (Table 2). All five circRNAs showed robust upregulation, which is consistent with the microarray findings (Figure 7). On their own, each of the five circRNAs showed the potential for differentiating HSCR tissue from normal tissue (Figure 8), and provided the following AUCs: hsa_circRNA_092493, 0.716; hsa_circRNA_101965, 0.951; hsa_circRNA_103118, 0.911; hsa_circRNA_103279, 0.738; and hsa_circRNA_104214, 0.929 .

\section{Discussion}

We herein performed what appears to be the first systematic circRNA profiling of HSCR, and we identified several circRNAs that may be useful as diagnostic markers that 
Table 2 Demographic and clinical characteristics in HSCR

\begin{tabular}{|c|c|c|}
\hline Characteristic & \multicolumn{2}{|c|}{ Patients included in the } \\
\hline $\mathrm{N}$ & 4 & 15 \\
\hline Male & 3 & 14 \\
\hline Female & 1 & 1 \\
\hline Vomiting & 2 & 13 \\
\hline Abdominal distension & 4 & 13 \\
\hline 24 hours without defecation & 2 & 6 \\
\hline Premature birth & 0 & 3 \\
\hline Gastrointestinal perforation & 1 & 1 \\
\hline Colitis & 1 & 5 \\
\hline Family medical history & 0 & 0 \\
\hline
\end{tabular}

Values are $\mathrm{n}$ or median [interquartile range]. HSCR, Hirschsprung's disease.

A

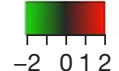

Row Z-score

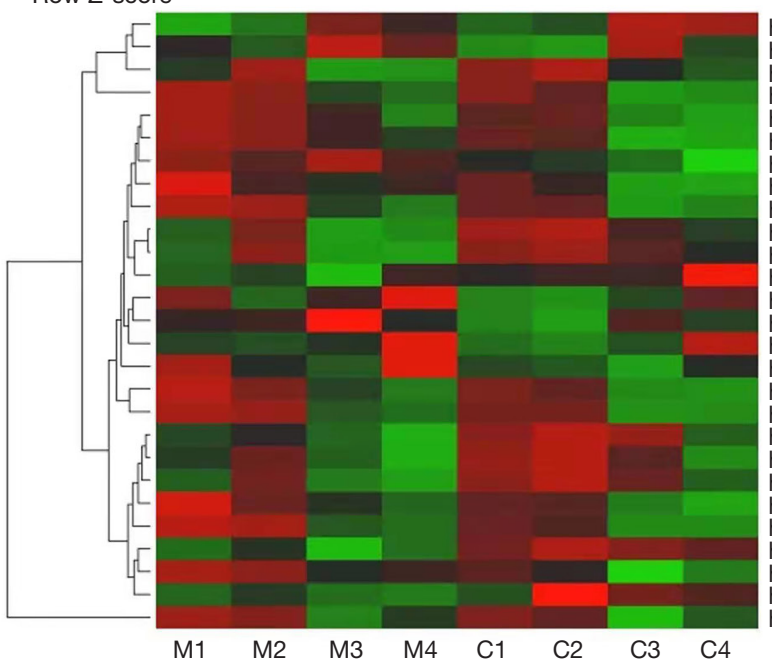

B

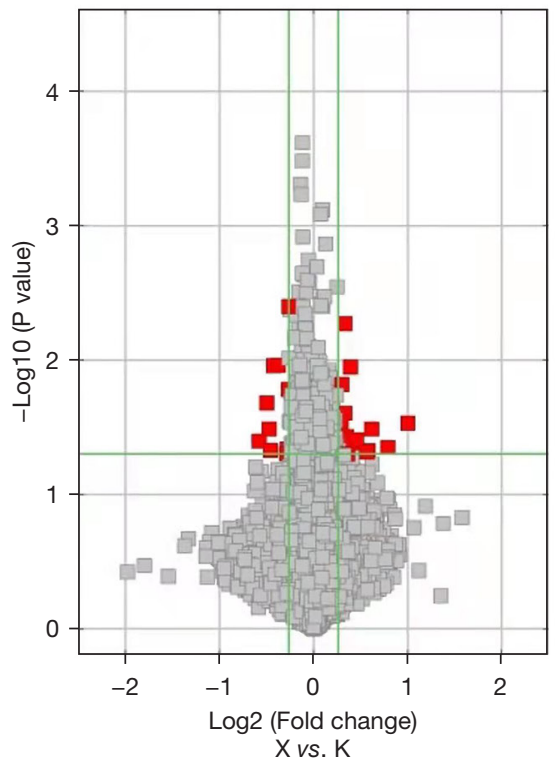

Figure 1 Differences in circRNA expression between HSCR and paired normal intestinal tissues. (A) Clustergram showing all of circRNA expression profiling of the samples. (B) Volcano plots showing differential expression of circRNAs. M, megacolon tissue; C, paired normal intestinal tissue; circRNA, circular RNA; HSCR, Hirschsprung's disease. 


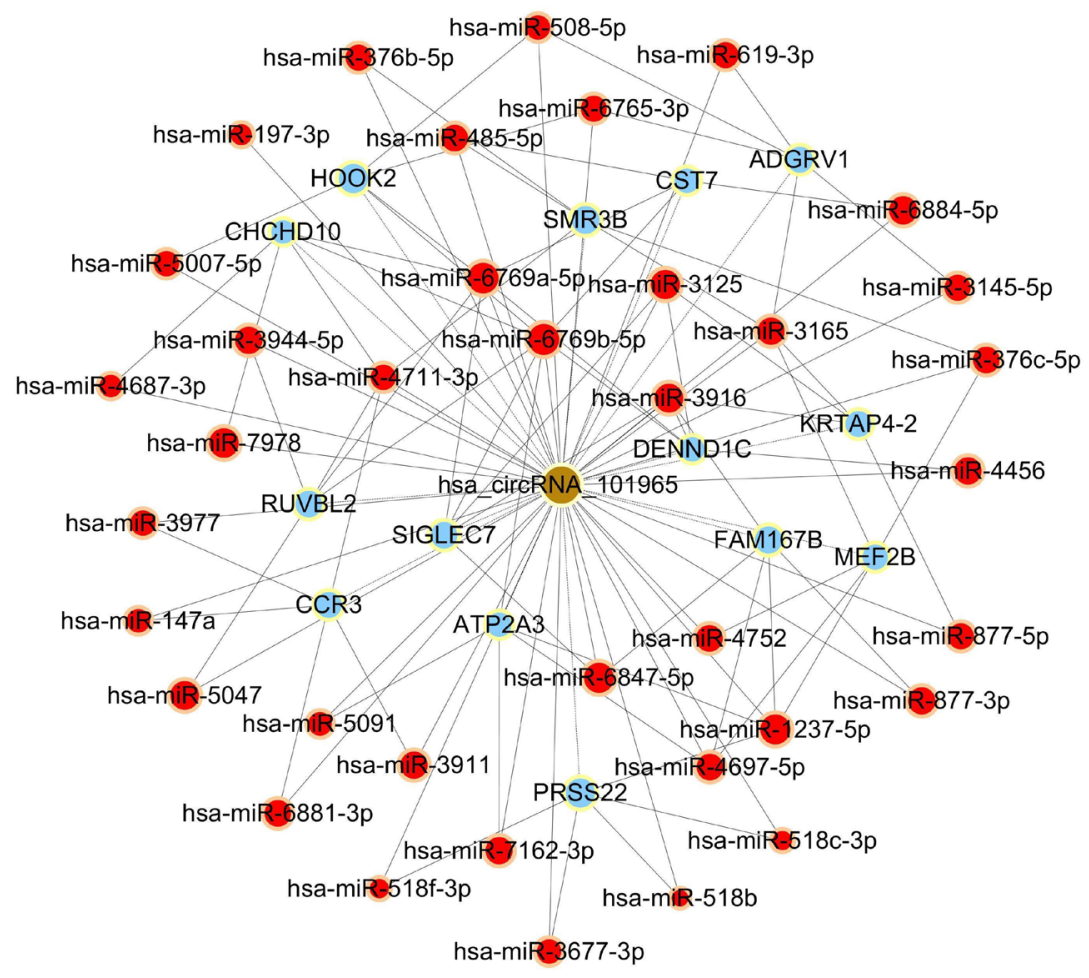

Figure 2 CircRNA-miRNA-mRNA regulatory network for hsa_circRNA_101965. circRNA, circular RNA.

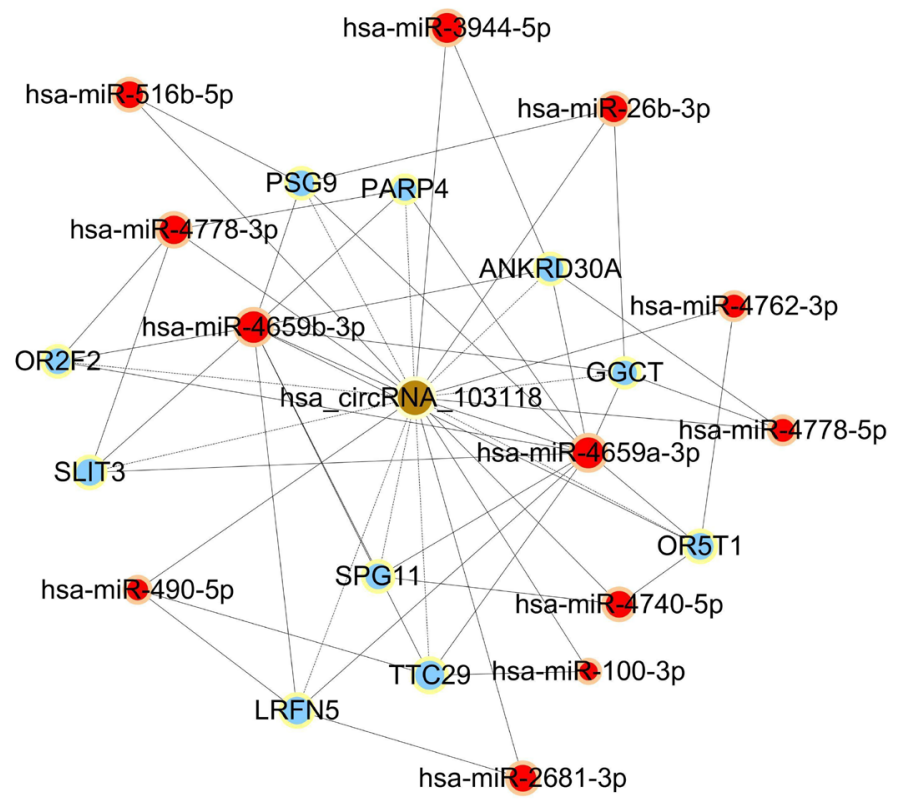

Figure 3 CircRNA-miRNA-mRNA regulatory network for hsa_circRNA_103118. circRNA, circular RNA. 


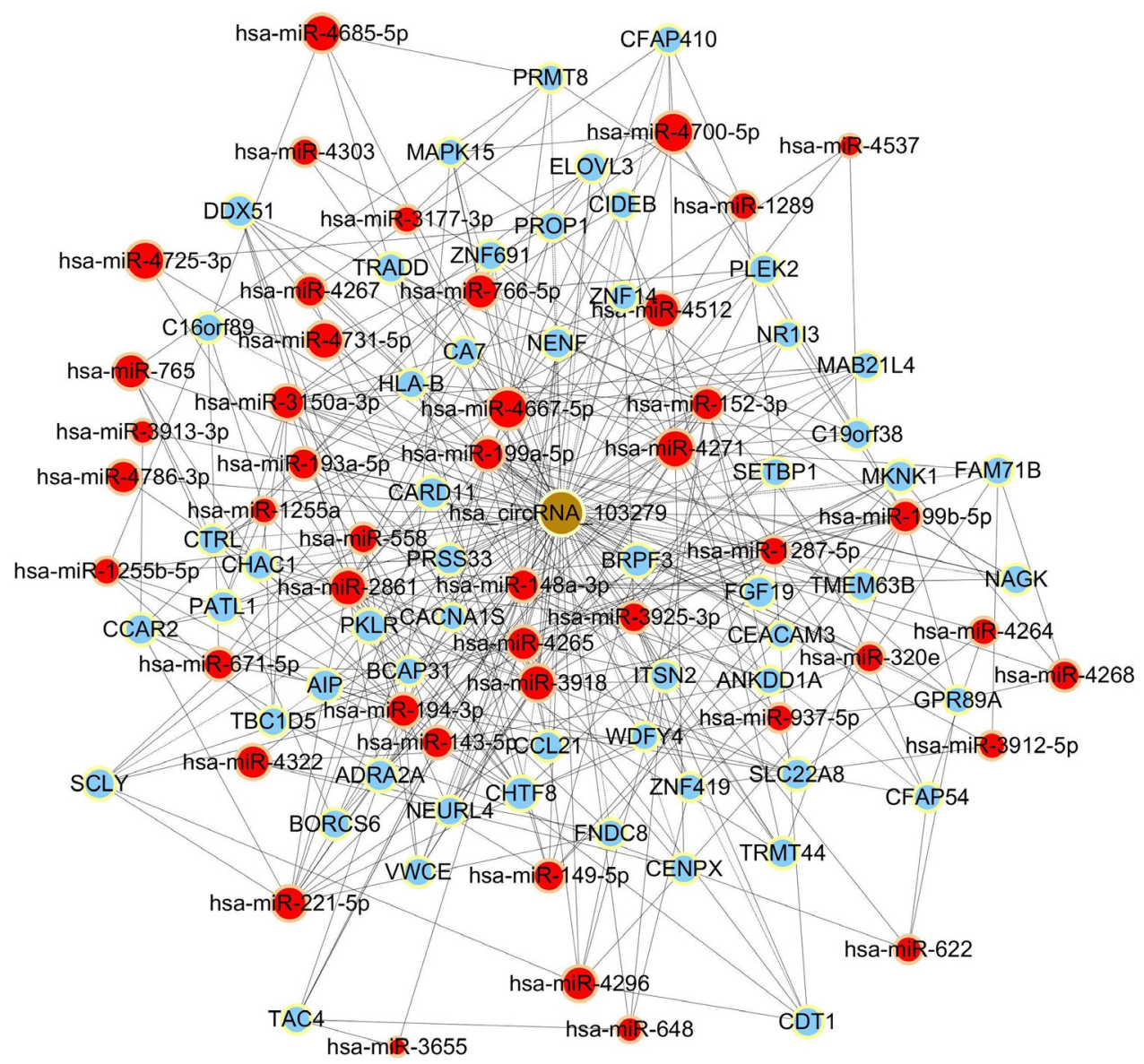

Figure 4 CircRNA-miRNA-mRNA regulatory network for hsa_circRNA_103279. circRNA, circular RNA.

might participate in molecular pathways. This work will thus improve our understanding of the disorder and allow us to devise appropriate therapeutic strategies. Our investigation builds upon several studies that implicate circRNAs in many aspects of biology and disease, including roles in cancer, heart disease, synaptic transmission, and aging $(21,22)$. Their abundance and stability in bodily fluids make them good candidates as biomarkers that can be assayed noninvasively. In addition, tissue-specific changes in circRNA expression have been associated with the initiation and progression of numerous diseases, including various kinds of cancers and cardiovascular and neurological diseases (23).

Some researchers have suggested that certain circRNAs play a critical role in the pathogenesis of HSCR $(13,24,25)$. Investigators have previously reported that three circRNAs, namely, circRNA-PRKCi, circRNA-CCDC66, and circRNA-ZNF609, acted as ceRNAs or sponges, and they primarily participated in the onset of HSCR via an increase in target genes by removing the inhibitory effect of miRNAs. In the present study, we revealed 27 dysregulated circRNAs in HSCR by microarray profiling, and we identified 5 representative circRNAs by verification experiments that entailed RT-PCR amplification. Our findings provide a comprehensive, genome-wide characterization of circRNAs in HSCR tissue and paired normal intestine. This then lays the foundation for extensive follow-up studies that will explore the expression of these circRNAs and their effects on the occurrence of HSCR. We constructed a circRNA-miRNA-mRNA regulatory network that highlighted several pathways that merit further study in order to elucidate the poorly understood pathology of HSCR, and we also identified therapeutic targets. We showed that each selected circRNA (containing at least one miRNA-binding site) was able to interact with several miRNAs (Figures 2-6). Consistent with our results, another 


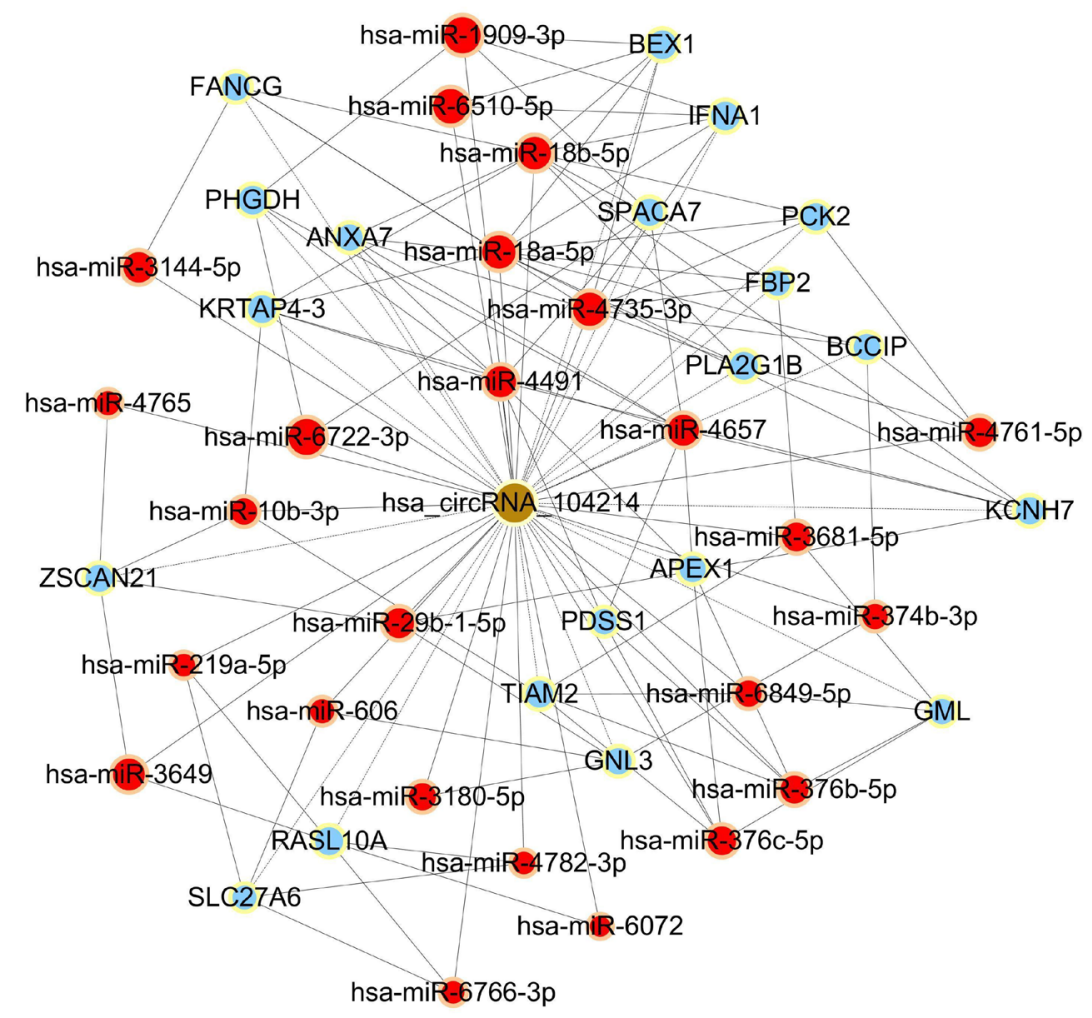

Figure 5 CircRNA-miRNA-mRNA regulatory network for hsa_circRNA_104214. circRNA, circular RNA.

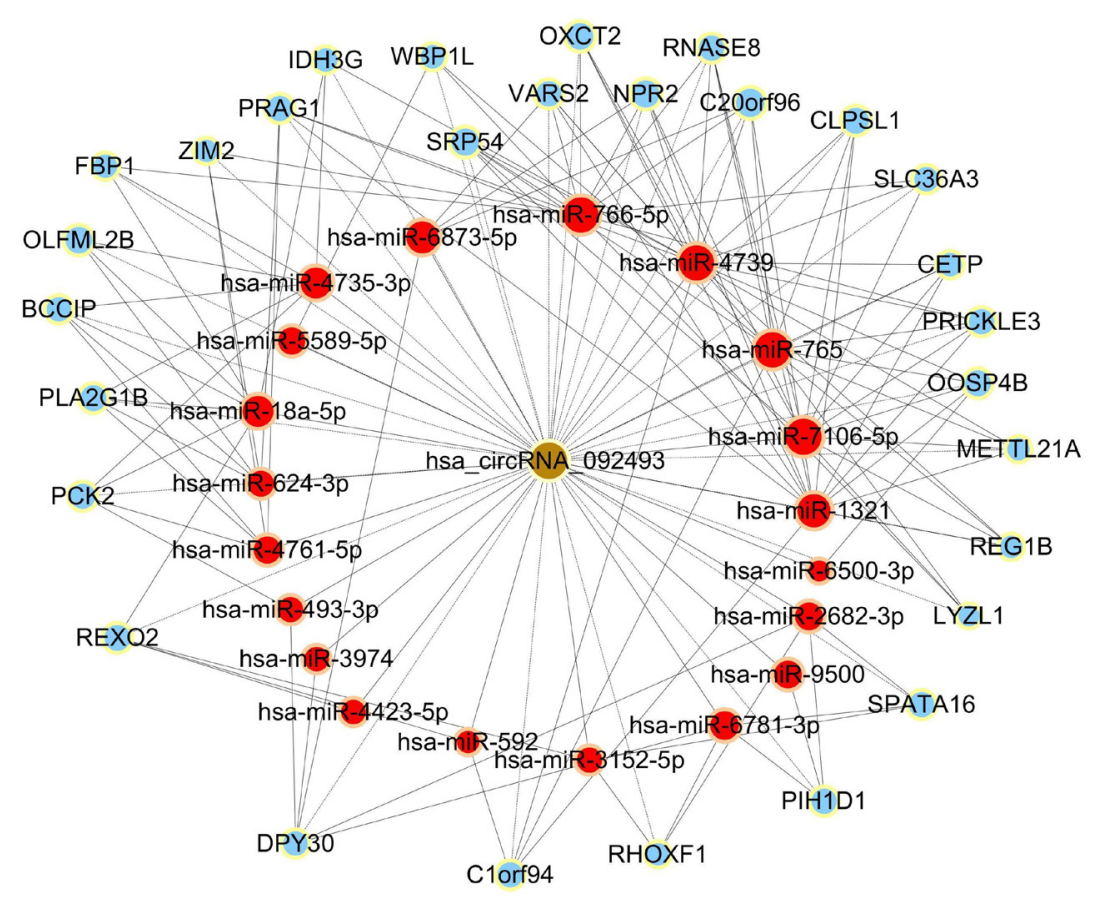

Figure 6 CircRNA-miRNA-mRNA regulatory network for hsa_circRNA_092493. circRNA, circular RNA. 

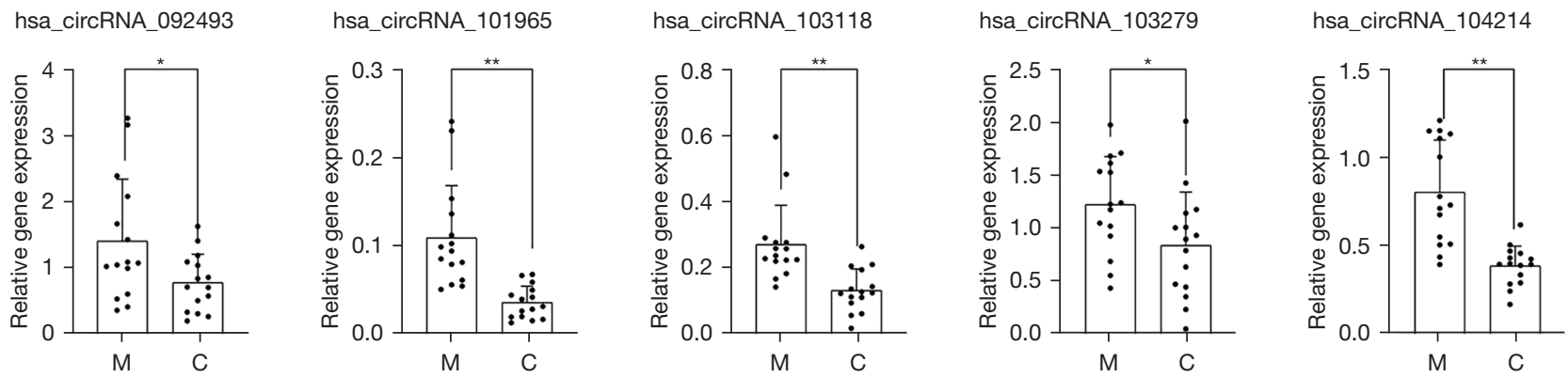

Figure 7 The expression levels of candidate circRNAs for validation in HSCR and paired normal intestinal tissue by qRT-PCR. The expression levels of candidate circRNAs that were strongly upregulated in HSCR than paired normal intestinal tissue. *, $\mathrm{P}<0.05$; **, $\mathrm{P}<0.01$. $M$, megacolon tissue; $\mathrm{C}$, paired normal intestinal tissue; circRNA, circular RNA; HSCR, Hirschsprung's disease.

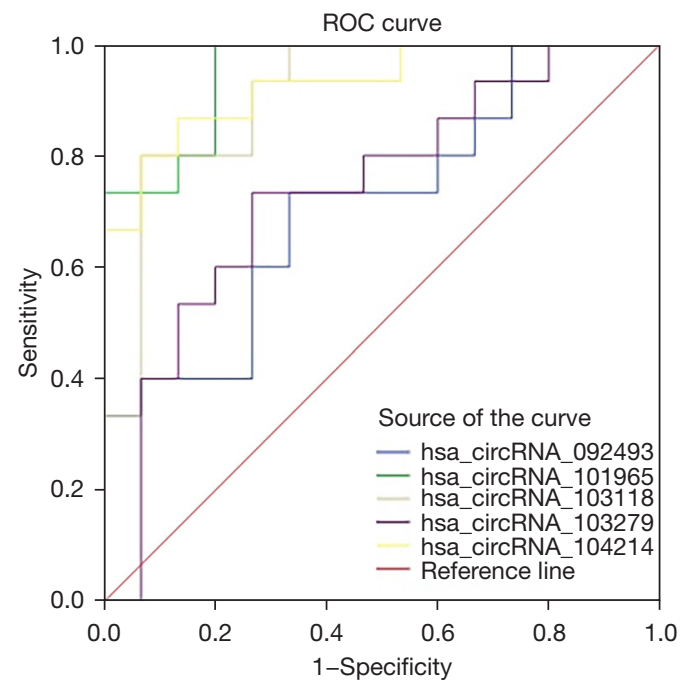

Figure 8 ROC curves of the five most strongly upregulated circRNAs in HSCR. ROC, receiver operating characteristic; circRNA, circular RNA; HSCR, Hirschsprung's disease.

study revealed that cir-CCDC66 can sponge miR-488 and induce expression of the target gene doublecortin (DCX), which may constitute a novel signaling pathway in the onset of HSCR (13). Additional experiments will be required in the future to evaluate the potential "housekeeping" role of these circRNAs and their effects on HSCR pathogenesis.

Previous work has suggested that miRNAs and long noncoding RNAs may be useful as biomarkers of HSCR (15), and the present study further indicates that circRNA, a third type of noncoding RNA, may also be valuable in this regard. In fact, the AUCs for the five most strongly upregulated circRNAs ranged from 0.72 to 0.95 , and combining multiple circRNAs may lead to even higher AUC values. Relative to other noncoding RNAs, circRNAs may be superior biomarkers as they persist longer in circulation, they are more conserved across species, and their expression differs for various diseases.

Our results should be interpreted carefully in light of several limitations. One is the relatively small sample size, and another is our reliance on a microarray that may lack the dynamic range required for appropriate detection. Since we only performed bioinformatics analyses, future biological experiments are necessary to verify and extend our findings.

\section{Conclusions}

We identified five circRNAs that were strongly up-regulated in HSCR: hsa_circRNA_092493, hsa_circRNA_101965, hsa_circRNA_103118, hsa_circRNA_103279, and hsa_ circRNA_104214. With these circRNAs, we constructed a circRNA-miRNA-mRNA regulatory network that will guide future work by clarifying the pathogenesis of this disorder and identifying appropriate therapeutic and diagnostic targets.

\section{Acknowledgments}

Funding: None.

\section{Footnote}

Reporting Checklist: The authors have completed the STARD reporting checklist. Available at https://tp.amegroups.com/ article/view/10.21037/tp-21-392/rc

Data Sharing Statement: Available at https://tp.amegroups. 
com/article/view/10.21037/tp-21-392/dss

Conflicts of Interest: All authors have completed the ICMJE uniform disclosure form (available at https://tp.amegroups. com/article/view/10.21037/tp-21-392/coif). The authors have no conflicts of interest to declare.

Ethical Statement: The authors are accountable for all aspects of the work in ensuring that questions related to the accuracy or integrity of any part of the work are appropriately investigated and resolved. The study was conducted in accordance with the Declaration of Helsinki (as revised in 2013). The study procedure was approved by the Ethics Committee of our Hospital (No. 20170606013) and written informed consent was obtained from the legal guardians of all of the participating children.

Open Access Statement: This is an Open Access article distributed in accordance with the Creative Commons Attribution-NonCommercial-NoDerivs 4.0 International License (CC BY-NC-ND 4.0), which permits the noncommercial replication and distribution of the article with the strict proviso that no changes or edits are made and the original work is properly cited (including links to both the formal publication through the relevant DOI and the license). See: https://creativecommons.org/licenses/by-nc-nd/4.0/.

\section{References}

1. Gershon MD. Developmental determinants of the independence and complexity of the enteric nervous system. Trends Neurosci 2010;33:446-56.

2. Ludman L, Spitz L, Tsuji H, et al. Hirschsprung's disease: functional and psychological follow up comparing total colonic and rectosigmoid aganglionosis. Arch Dis Child 2002;86:348-51.

3. Mills JL, Konkin DE, Milner R, et al. Long-term bowel function and quality of life in children with Hirschsprung's disease. J Pediatr Surg 2008;43:899-905.

4. Minford JL, Ram A, Turnock RR, et al. Comparison of functional outcomes of Duhamel and transanal endorectal coloanal anastomosis for Hirschsprung's disease. J Pediatr Surg 2004;39:161-5; discussion 161-5.

5. Tam PK. Hirschsprung's disease: A bridge for science and surgery. J Pediatr Surg 2016;51:18-22.

6. Muto M, Matsufuji H, Taguchi T, et al. Japanese clinical practice guidelines for allied disorders of Hirschsprung's disease, 2017. Pediatr Int 2018;60:400-10.
7. Ambartsumyan L, Smith C, Kapur RP. Diagnosis of Hirschsprung Disease. Pediatr Dev Pathol 2020;23:8-22.

8. Putnam LR, John SD, Greenfield SA, et al. The utility of the contrast enema in neonates with suspected Hirschsprung disease. J Pediatr Surg 2015;50:963-6.

9. Rybak-Wolf A, Stottmeister C, Glažar P, et al. Circular RNAs in the Mammalian Brain Are Highly Abundant, Conserved, and Dynamically Expressed. Mol Cell 2015;58:870-85.

10. Li CY, Ma L, Yu B. Circular RNA hsa_circ_0003575 regulates oxLDL induced vascular endothelial cells proliferation and angiogenesis. Biomed Pharmacother 2017;95:1514-9.

11. Piwecka M, Glažar P, Hernandez-Miranda LR, et al. Loss of a mammalian circular RNA locus causes miRNA deregulation and affects brain function. Science 2017;357:eaam8526.

12. Guarnerio J, Bezzi M, Jeong JC, et al. Oncogenic Role of Fusion-circRNAs Derived from Cancer-Associated Chromosomal Translocations. Cell 2016;165:289-302.

13. Wen Z, Shen Q, Zhang H, et al. Circular RNA CCDC66 targets DCX to regulate cell proliferation and migration by sponging miR-488-3p in Hirschsprung's disease. J Cell Physiol 2019;234:10576-87.

14. Peng L, Chen G, Zhu Z, et al. Correction: Circular RNA ZNF609 functions as a competitive endogenous RNA to regulate AKT3 expression by sponging miR-150-5p in Hirschsprung's disease. Oncotarget 2019;10:3313-4.

15. Sergi CM, Caluseriu O, McColl H, et al. Hirschsprung's disease: clinical dysmorphology, genes, micro-RNAs, and future perspectives. Pediatr Res 2017;81:177-91.

16. Liu W, Zhang J, Zou C, et al. Microarray Expression Profile and Functional Analysis of Circular RNAs in Osteosarcoma. Cell Physiol Biochem 2017;43:969-85.

17. Lin SP, Ye S, Long Y, et al. Circular RNA expression alterations are involved in OGD/R-induced neuron injury. Biochem Biophys Res Commun 2016;471:52-6.

18. Zhou J, Xiong Q, Chen H, et al. Identification of the Spinal Expression Profile of Non-coding RNAs Involved in Neuropathic Pain Following Spared Nerve Injury by Sequence Analysis. Front Mol Neurosci 2017;10:91.

19. Tay Y, Rinn J, Pandolfi PP. The multilayered complexity of ceRNA crosstalk and competition. Nature 2014;505:344-52.

20. Xie L, Mao M, Xiong K, et al. Circular RNAs: A Novel Player in Development and Disease of the Central Nervous System. Front Cell Neurosci 2017;11:354.

21. Li S, Sun X, Miao S, et al. hsa_circ_0000729, a potential prognostic biomarker in lung adenocarcinoma. Thorac 
Cancer 2018;9:924-30.

22. Li P, Yang X, Yuan W, et al. CircRNA-Cdr1as Exerts AntiOncogenic Functions in Bladder Cancer by Sponging MicroRNA-135a. Cell Physiol Biochem 2018;46:1606-16.

23. Qu S, Yang X, Li X, et al. Circular RNA: A new star of noncoding RNAs. Cancer Lett 2015;365:141-8.

24. Peng L, Chen G, Zhu Z, et al. Circular RNA ZNF609

Cite this article as: Huang SG, Cheng Y, Li D, Sun C, Fang F, Guo WL. Systematic screen of potential circular RNA biomarkers of Hirschsprung's disease. Transl Pediatr 2022;11(1):10-19. doi: 10.21037/tp-21-392 functions as a competitive endogenous RNA to regulate AKT3 expression by sponging miR-150-5p in Hirschsprung's disease. Oncotarget 2017;8:808-18.

25. Zhou L, Li Y, Jiang W, et al. Down-regulation of circPRKCI inhibits cell migration and proliferation in Hirschsprung disease by suppressing the expression of miR-1324 target PLCB1. Cell Cycle 2018;17:1092-101. 